\title{
Abdominal aortic aneurysm repair: long-term follow-up of endovascular versus open repair
}

\author{
Gabriele Piffaretti ${ }^{1}$, Giovanni Mariscalco ${ }^{2}$, Francesca Riva ${ }^{3}$, Federico Fontana ${ }^{4}$, \\ Gianpaolo Carrafiello ${ }^{4}$, Patrizio Castelli ${ }^{1}$
}

\begin{abstract}
${ }^{1}$ Vascular Surgery, Department of Surgery and Morphological Sciences Circolo University Hospital, University of Insubria School of Medicine, Varese, Italy ${ }^{2}$ Cardiac Surgery, Department of Surgery and Morphological Sciences Circolo University Hospital, University of Insubria School of Medicine, Varese, Italy ${ }^{3}$ Vascular Surgery, Civic Hospital, Legnano, Italy

${ }^{4}$ Interventional Radiology, Department of Radiology Circolo University Hospital, University of Insubria School of Medicine, Varese, Italy
\end{abstract}

Submitted: 2 September 2012

Accepted: 7 March 2013

Arch Med Sci 2014; 10, 2: 273-282

DOI: 10.5114 /aoms.2014.42579

Copyright (C) 2014 Termedia \& Banach
Corresponding author: Gabriele Piffaretti MD PhD Vascular Surgery Department of Surgery and Morphological Sciences Circolo University Hospital University of Insubria School of Medicine Via Guicciardini, 9 21100 Varese, Italy Phone: +390332 393.259 Fax: +390332 278.581 E-mail: gabriele.piffaretti@ uninsubria.it

\section{Abstract}

Introduction: To compare early and long-term outcomes of endovascular abdominal aortic aneurysm repair (EVAR) versus open repair (OPEN). Design: Prospective observational, per protocol, non-randomized, with retrospective analyses.

Material and methods: Between 2000 and 2005, a total of 311 patients having EVAR or OPEN repair of infrarenal abdominal aortic aneurysms were identified and included in this prospective single-center observational study. A propensity score-based optimal-matching algorithm was employed, and 138 patients undergoing EVAR procedures were matched $(1: 1)$ to OPEN repair.

Results: Open repair showed higher hospital mortality (17\% vs. $6 \%, p=0.004)$, respiratory failure $(p<0.026)$, transfusion requirement $(p<0.001)$, and intensive care unit admission ( $27 \%$ vs. $7 \%, p<0.001)$, and longer hospitalization $(p<0.001)$. Median follow-up was 70 months $\left(25^{\text {th }}\right.$ to $75^{\text {th }}$ percentile, 24 to 101). Actuarial survival estimates at 1, 5 and 10 years were $93 \%, 74 \%$, $49 \%$ for the OPEN group compared to $89 \%, 69 \%, 59 \%$ for the EVAR group $(p=0.465)$. A significant difference between groups was observed in younger patients $(<75$ years) only $(p<0.044)$. Late complication and re-intervention rates were significantly higher in EVAR patients $(p<0.001$ and $p=0.002$, respectively). Freedom from late complications at 1,5 and 10 years was $96 \%, 92 \%, 86 \%$, and $84 \%, 70 \%, 64 \%$ for OPEN and EVAR procedures, respectively.

Conclusions: Our experience confirms the excellent results of the EVAR procedures, offering excellent early and long-term results in terms of safety and reduction of mortality. Patients $<75$ years seem to benefit from EVAR not only in the immediate postoperative period but even in a long-term perspective.

Key words: abdominal aortic aneurysms, long-term results, aneurysm mortality.

\section{Introduction}

Since the beginning of its application, endovascular aortic repair (EVAR) has been considered for abdominal aortic aneurysms with suit- 
able anatomy in higher risk patients only [1-5]. Thereafter, the promising results coming from single-center series extended the application of EVAR even in challenging cases [6-8].

Recently, the published long-term results of celebrated clinical trials have questioned the benefits of EVAR by reporting the high need of secondary interventions $[9,10]$. Nevertheless, the same studies also confirmed low perioperative mortality and morbidity rates for EVAR, and low reintervention rates were observed for favorable anatomy. Most of the redo procedures were amenable to endovascular or minimally invasive treatments, with a lower negative impact on the long-term outcomes [6, 7, 10, 11].

Few contemporary studies have reported a comparison of late outcomes after either EVAR or conventional open repair (OPEN) of abdominal aortic aneurysms (AAA) [1-14]. The present study aimed to compare the clinical and technical outcomes between EVAR and conventional OPEN procedures.

\section{Material and methods}

This was a prospective, observational, non-randomized, per protocol study designed to compare long-term results of EVAR vs. OPEN repair for $a b$ dominal aortic aneurysms.

\section{Patient population}

Patients who had undergone repair of an infrarenal ruptured AAA were included. Inclusion criteria were $A A A \geq 55 \mathrm{~mm}$ for male ( $50 \mathrm{~mm}$ for female) patients or diameter increase of $\geq 5 \mathrm{~mm}$ on two consecutive follow-up examinations. Symptomatic and ruptured AAA were included to have "real life" analyses for either OPEN or EVAR treatments. Conversely, subjects with redo aortic surgery or suprarenal/thoraco-abdominal aortic reconstruction were excluded. The study protocol was in compliance with the local Institutional Review Board and received full approval. All patients gave their consent to participate.

\section{Patient management}

Patients were selected as possible candidates for EVAR on the basis of contrast enhanced computed tomography (CT) with 3-mm to 5-mm cuts. Three-dimensional vascular reconstructions were performed when possible. Risk factors and morphologic features were classified according to the Society for Vascular Surgery/International Society for Cardiovascular Surgery reporting standards $[15,16]$. Vascular surgeons and interventional radiologists were responsible for selecting OPEN versus EVAR for each patient: CR was always offered in patients with unsuitable anatomy; however, anatomic criteria for exclusion from EVAR were less stringent in patients at high risk, but in the first phase of the endovascular experience, EVAR was also offered to low-risk patients with suitable anatomy who preferred this type of intervention. All procedures were performed in the theater, fully equipped for all types of endovascular procedures; radiology imaging was performed with a high-quality portable C-arm (BV300 ${ }^{\circledR}$-Siemens; Munich-GER) fluoroscopic unit with digital imaging and road mapping capability. Conventional repair (OPEN group) was performed under general anesthesia, while EVAR procedures were performed under general anesthesia with oro-tracheal intubation (in the great majority of cases) or under epidural anesthesia or local anesthesia in the remaining patients. Open common femoral artery exposure by means of a small groin incision was used for the endograft (EG) access. A tube graft was used in 109 (79\%) CR cases; generally, a knitted Dacron ( $n=136,99 \%)$ graft (Uni-graft ${ }^{\oplus}$, Carbograft ${ }^{\oplus}$; Bard Inc.-Murray Hill-NJ; USA) was implanted. Endograft configuration was bifurcated infrarenal in 124 (71.7\%) cases, bifurcated transrenal in 30 (17.3\%), aortouni-iliac in 18 (10.4\%), and tube transrenal in 1 (0.6\%). Manufacturers were as follows: Excluder ${ }^{\circledR}$ (W.L. Gore \& Associates; Flagstaff, AZ; USA) for all infrarenal EG, transrenal EG used were Zenith $^{\circledR}$ (Cook Inc.; Bloomington, IN; USA) in 42, Talent ${ }^{\circledR}$ (Medtronic; Santa Rosa, CA; USA) in 6, Lifepath $^{\circledast}$ (Edwards; Irvine, CA; USA) in 4, Powerlink ${ }^{\circledR}$ (Endologix; Irvine, CA; USA) in 3, and Fortron ${ }^{\circledast}$ (Cordis Corp; Miami Lakes, FL; USA) in 1 case.

The postoperative surveillance protocol consisted of clinical, duplex ultrasound scanning at 1 , 6, 12 months, and then on a yearly basis after CR. Computed tomography scans were performed only when graft-related complications were suspected. Patients who underwent EVAR were scheduled for routine clinical follow-up with CT scheduled at 1, 6 and 12 months, then yearly.

\section{Outcome measures and definitions}

Primary outcomes included operative ( $\leq 30$ days) mortality, AAA rupture, aneurysm-related mortality, surgical conversion to open conventional repair, and late survival. Aneurysm-related mortality (ARM) was defined as death from any cause within 30 days of the primary EVAR procedure, death within 30 days of any secondary reintervention or surgical conversion, or any death due to aneurysm rupture or device complication. In addition, secondary outcomes including data related to endoleak and EG patency were examined. Finally, the frequency of secondary reintervention was determined, as well as the time, method, and success of such re-interventions. Outcome criteria were defined according to the Ad Hoc Committee 
for Standardized Reporting Practices in Vascular Surgery of the Society for Vascular Surgery/American Association for Vascular Surgery (SVS/AAVS) $[15,16]$.

\section{Statistical analysis}

Clinical data were prospectively recorded and tabulated with Microsoft Excel (Microsoft ${ }^{\oplus}$ Corp, Redmond, Washington). Data were described as mean and standard deviation (SD) or median and interquartile range $\left(25^{\text {th }}\right.$ to $75^{\text {th }}$ percentile) for continuous variables and as number and/or percent for the categorical ones. Continuous variables were tested for normal distribution by the Kolmogorov-Smirnov test and compared between groups with unpaired Student $t$ test for normally distributed values; otherwise, the Mann-Whitney $U$ test was used. In the case of dichotomous variables, group differences were examined by Pearson $\chi^{2}$ or Fisher exact tests as appropriate. In an attempt to control for selection bias related to the procedures of AAA repair, a propensity score analysis was developed, assuming the OPEN group as the treatment one. A multivariable logistic regression analysis to calculate propensity score was applied. The propensity score was based on significant demographic and clinical variables in the univariate analysis. The variables included in the final model were age, gender, emergency/elective status, prior vascular surgery (reoperation), history of coronary artery disease, congestive heart failure, concomitant heart valvulopathy, cardiac arrhythmias, hypertension, diabetes mellitus, dyslipidemia, chronic obstructive pulmonary disease, history of cerebrovascular accident, chronic renal failure, aneurysm presentation, SVS score, and finally the aneurysm diameter. This process generated a propensity score between 0 and 1 and patients receiving EVAR procedures were matched 1 : 1 to those undergoing conventional repair (OPEN group), using the Rosenbaum optimal matching algorithm [17]. This approach minimizes the overall distance between observations and was conducted using Mahalanobis distance within propensity score calipers (no matches outside the calipers) [17]. After the propensity score match was performed, we assessed differences between the two groups as above. Absolute standardized differences were estimated to evaluate the prematch imbalance and postmatch balance [17]. An absolute standardized difference of $0 \%$ indicates no residual bias and differences $<10 \%$ are considered inconsequential [18].

Univariate and multivariate approaches (logistic and Cox regression analyses) were then performed to identify variables potentially associated with study end-points. Regarding multivariate analy- ses, a stepwise approach was used and confirmed by backward and forward methods. The models were built using variables that demonstrated a $p$ value $<0.20$ in univariate mode. The significance within the models was evaluated with the Wald test. The strength of the association of variables with hospital mortality was estimated by calculating the odds ratio (OR) and 95\% confidence intervals $(\mathrm{Cls})$. The model was calibrated by the Hosmer-Lemeshow goodness-of-fit test, as well as residual diagnostics (deviance and dfBetas); model discrimination was evaluated by using the area under the receiver operating characteristic (ROC) curve. For late outcomes (log-term mortality and morbidity, freedom from reinterventions and complications), hazard ratios (HRs) were generated by a Cox regression analysis. Late outcomes were also assessed using Kaplan-Meier life-table analysis and the Mantel-Cox log-rank test was applied when comparing subgroups. All tests were 2 sided, and a value of $p \leq 0.05$ was considered statistically significant. Statistical analysis was computed using SPSS, release 19.0 for Windows (SPSS ${ }^{\circledR}$ Inc; Chicago, IL; USA) and NCSS 2007, release 7.1 (Kaysville, UT; USA).

\section{Results}

\section{Patient demographics}

Between January 2000 and December 2005, a total of 311 patients having EVAR or OPEN repair of infrarenal AAA treated in our tertiary care university hospital were identified and formed the study group. Among the 311 enrolled patients, repair was performed on an emergency basis in $70(23 \%)$ cases. The study cohort contained 279 (90\%) males; mean age was $71.7 \pm 8.9$ years (range: 48-96). OPEN repair was performed in 138 (44\%) cases, EVAR in 173 (56\%). The propensity score-based greedy-matching algorithm matched 138 patients for each group (Table I). Postmatch absolute standardized differences for all measured covariates were $<10 \%$ (most were $<5 \%$ ), suggesting substantial covariate balance across groups (Figure 1). Both groups appeared comparable in pre- and perioperative characteristics and risk factors; mean SVS score was not statistically significant (3 $\left(25^{\text {th }}\right.$ to $75^{\text {th }}$ percentile, 2 to 5$)$ vs. 3 ( $25^{\text {th }}$ to $75^{\text {th }}$ percentile, 2 to 4$), p=0.487$ ).

\section{In-hospital outcome}

The primary technical success (successful deployment with no complication requiring adjunctive procedures) rate was 99.6\% (275/276); conversion from EVAR to OPEN repair was never needed. Mean operation time was longer in the OPEN group (150 $\mathrm{min}$ ( $25^{\text {th }}$ to $75^{\text {th }}$ percentile, 120 to $196 \mathrm{~min})$ vs. $120 \mathrm{~min}$ ( $25^{\text {th }}$ to $75^{\text {th }}$ percentile, 
Table I. Demographic and clinical data with reference to procedure group

\begin{tabular}{|c|c|c|c|c|c|}
\hline \multirow[t]{2}{*}{ Variable* } & \multicolumn{2}{|c|}{ Prematch } & \multicolumn{3}{|c|}{ Postmatch } \\
\hline & $\operatorname{EVAR}(n=173)$ & $P^{t, \neq}$ & $\operatorname{EVAR}(n=138)$ & OPEN $(n=138)$ & $P^{\dagger}$ \\
\hline Age [years] & $72.2 \pm 8.5$ & 0.302 & $71.5 \pm 8.6$ & $71.2 \pm 9.3$ & 0.742 \\
\hline Males & $157(90.8)$ & 0.499 & $125(90.6)$ & $122(88.4)$ & 0.556 \\
\hline Emergency & $44(25.4)$ & 0.167 & 27 (19.6) & $26(18.8)$ & 0.879 \\
\hline Prior surgery & $25(14.5)$ & 0.119 & $15(10.9)$ & $12(8.7)$ & 0.543 \\
\hline CAD & $60(34.7)$ & 0.178 & $40(29.0)$ & $38(27.5)$ & 0.789 \\
\hline Arrhythmia & $41(23.7)$ & 0.091 & $25(18.1)$ & $22(15.9)$ & 0.631 \\
\hline $\mathrm{CHF}$ & $15(8.7)$ & 0.035 & $6(4.3)$ & $4(2.9)$ & 0.749 \\
\hline Heart valvulopathy & $9(5.2)$ & 0.621 & $9(6.5)$ & & \\
\hline Hypertension & $138(79.8)$ & 0.353 & $105(76.1)$ & $104(75.4)$ & 0.888 \\
\hline Diabetes & $17(9.8)$ & 0.296 & $11(8.0)$ & $9(6.5)$ & 0.642 \\
\hline Dyslipidemia & $54(31.2)$ & 0.480 & $43(31.2)$ & $38(27.5)$ & 0.509 \\
\hline COPD & $22(12.7)$ & 0.070 & $12(8.7)$ & $9(6.5)$ & 0.496 \\
\hline CVA & $21(12.1)$ & 0.728 & $18(13.0)$ & $15(10.9)$ & 0.578 \\
\hline CRF & $16(9.2)$ & 0.094 & $6(4.3)$ & $6(4.3)$ & 0.999 \\
\hline SVS Score $(n)$ & $4(3-5)$ & 0.035 & $3(2-5)$ & $3(2-4)$ & 0.487 \\
\hline Presentation: & & 0.088 & & & 0.331 \\
\hline Asymptomatic & $134(77.5)$ & & $112(81.2)$ & $110(79.7)$ & \\
\hline Symptomatic & $15(8.7)$ & & $8(5.8)$ & $4(2.9)$ & \\
\hline Ruptured & $24(13.9)$ & & $18(13.0)$ & $24(17.4)$ & \\
\hline Aneurysm diameter [mm] & $73(66-78)$ & 0.001 & $50(47-60)$ & $50(40-60)$ & 0.322 \\
\hline
\end{tabular}

${ }^{*}$ For continuous variables, mean $\pm S D$ or median $\left(25^{\text {th }}\right.$ to $75^{\text {th }}$ percentile); for categorical variables, number (percent); ${ }^{\dagger}$ for continuous variables, Student $t$ test or the Mann-Whitney $U$ test; for categorical ones, $\chi^{2}$ or Fisher exact test; ${ }^{\ddagger} E V A R$ compared with OPEN procedures in whole patient population (prematch comparison); CAD - coronary artery disease, CHF - chronic heart failure, COPD - chronic obstructive pulmonary disease, CRF - chronic renal failure, CVA - cerebrovascular accidents, SVS - Society for Vascular Surgery

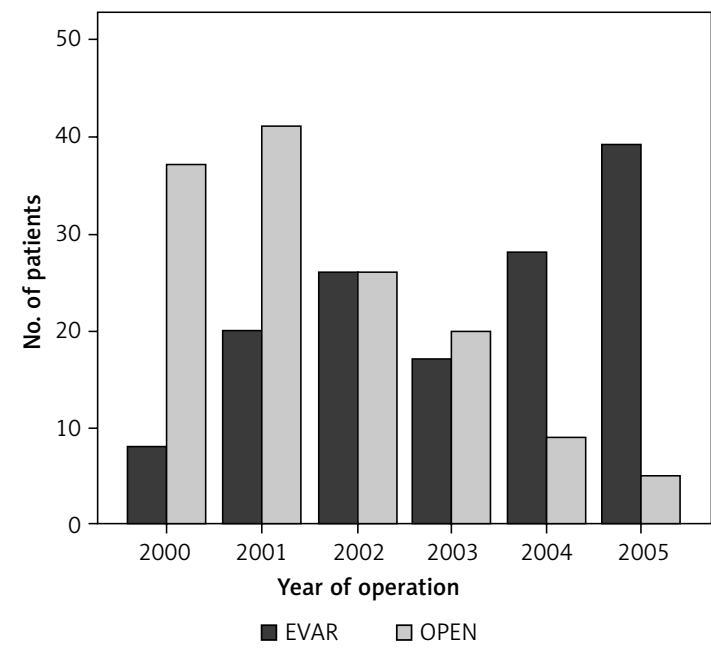

Figure 1. Baseline risk factors and variables: Absolute standardized differences between OPEN (conventional surgical repair) and EVAR (endovascular aortic repair) for abdominal aortic aneurysm, in the aggregate (black open diamonds) and in the propensity matched cohorts (gray closed diamonds)
90 to $142 \mathrm{~min}), p<0.001)$. Adjunctive procedures were performed similarly (17\% vs. $20 \%, p=0.639$ ).

Postoperatively, the OPEN group had a higher complication rate in terms of respiratory failure occurrence $(12 \%$ vs. $4 \%, p<0.026)$ and transfusion requirement ( $46 \%$ vs. $19 \%, p<0.001)$ (Table II). The intensive care unit (ICU) admission rate $(27 \%$ vs. $7 \%, p<0.001$ ) was higher in the OPEN group; a longer hospitalization ( 7 days $\left(25^{\text {th }}\right.$ to $75^{\text {th }}$ percentile, 8 to 12 days) vs. 5 days $\left(25^{\text {th }}\right.$ to $75^{\text {th }}$ percentile, 4 to 7 days), $p<0.001$ ) was observed in the OPEN group.

The overall in-hospital mortality in these two groups was $11 \%$ (31/276 patients). In-hospital mortality was higher in older (75.9 \pm 9.5 vs. 70.8 \pm 8.7 years, $p=0.006$ ) patients; they also had more co-morbidities (SVS score: $4.2 \pm 1.9$ vs. 3.3 $\pm 1.6, p=0.020)$. Hospital mortality was higher in the OPEN group ( $17 \%$ vs. $6 \%, p=0.004)$. Although this difference persisted between groups in patients aged $\geq 75$ years ( $23 \%$ vs. $7 \%, p=0.033$ ), 
Table II. Postoperative data with reference to procedure group

\begin{tabular}{|c|c|c|c|}
\hline Variable* & EVAR $(n=138)$ & OPEN $(n=138)$ & $P^{\dagger}$ \\
\hline \multicolumn{4}{|l|}{ Operative: } \\
\hline Adjunctive procedure & $27(19.6)$ & $23(16.7)$ & 0.532 \\
\hline Operation time [min] & $120(90-142)$ & $150(120-196)$ & $<0.001$ \\
\hline \multicolumn{4}{|l|}{ Postoperative: } \\
\hline Reoperation: & & & 0.112 \\
\hline Endovascular & $2(1.4)$ & $3(2.2)$ & \\
\hline Conventional surgery & $5(3.6)$ & $11(8.0)$ & \\
\hline Transfusion: & $26(18.8)$ & $64(46.4)$ & $<0.001$ \\
\hline RBC units $(n)$ & $0.7 \pm 1.7$ & $2.0 \pm 2.4$ & $<0.001$ \\
\hline FFP units $(n)$ & $0.7 \pm 1.7$ & $1.9 \pm 2.4$ & $<0.001$ \\
\hline Complications: & $21(15.2)$ & $32(23.2)$ & 0.093 \\
\hline Cardiac & $5(3.6)$ & $11(8.0)$ & 0.197 \\
\hline Respiratory & $6(4.3)$ & $16(11.6)$ & 0.026 \\
\hline Renal & $5(3.6)$ & $3(2.2)$ & 0.502 \\
\hline Procedure related & $9(6.5)$ & $13(9.4)$ & 0.374 \\
\hline ICU admission & $9(6.5)$ & $37(26.8)$ & $<0.001$ \\
\hline LOS (days) & $5(4-7)$ & $7(8-12)$ & $<0.001$ \\
\hline Hospital mortality & $8(5.8)$ & $23(16.7)$ & 0.004 \\
\hline
\end{tabular}

${ }^{*}$ For continuous variables, mean $\pm S D$ or median $\left(25^{\text {th }}\right.$ to $75^{\text {th }}$ percentile); for categorical variables, number (percent); ${ }^{\dagger}$ For continuous variables, Student $t$ test or the Mann-Whitney $U$ test; for categorical variables, Pearson's $\chi^{2}$ or Fisher exact test; ICU - intensive care unit, $L O S$ - length of hospitalization, RBC - red blood cells, FFP - fresh frozen plasma

no differences were noted for patients aged $<75$ and OPEN procedure $(p \leq 0.001, p=0.003$, and years $(12 \%$ vs. $5 \%, p=0.100)$. In multivariable $p=0.009$, respectively; Table III). The Hosmer-Leanalysis, predictors of hospital mortality were meshow goodness-of-fit test was not significant clinical presentations of AAA, chronic heart failure for lack of fit $\left(\chi^{2}[3 \mathrm{df}]=1.97, p=0.602\right)$, indicating

Table III. Independent predictors for hospital mortality, late mortality and reinterventions

\begin{tabular}{|lcccc|}
\hline Variable & Wald $\chi^{2}$ & $P$ & OR $^{*}$ & \\
\hline \begin{tabular}{l} 
Hospital mortality \\
\hline Clinical presentation ${ }^{\dagger}:$
\end{tabular} & 31.98 & & & \\
\hline AAA & 8.16 & 0.001 & \\
\hline \multicolumn{1}{c}{ Ruptured AAA } & 22.03 & 0.004 & 17.93 & $2.48-129.83$ \\
\hline CHF & 8.78 & 0.001 & 14.57 & $4.76-44.62$ \\
\hline OPEN procedure & 6.82 & 0.003 & 31.87 & $3.23-314.58$ \\
\hline Late mortality: & & & 4.86 & $1.48-15.93$ \\
\hline Age & 37.47 & $<0.001$ & 1.09 & $1.06-1.12$ \\
\hline CHF & 22.82 & $<0.001$ & 7.34 & $3.24-16.61$ \\
\hline ICU admission & 5.80 & 0.016 & 2.04 & $1.14-3.64$ \\
\hline Transfusion & 4.62 & 0.032 & 1.64 & $1.04-2.57$ \\
\hline Late reintervention: & & & 1.03 & $1.01-1.05$ \\
\hline \begin{tabular}{l} 
Aneurysm diameter \\
\hline EVAR procedure
\end{tabular} & 9.64 & 0.002 & 2.95 & $1.43-6.12$ \\
\hline History of CVA & 8.48 & 0.004 & 2.90 & $1.39-6.05$ \\
\hline
\end{tabular}

${ }^{\star}$ For hospital mortality multivariable logistic regression; for late mortality and reinterventions, multivariable Cox analysis; $A A A-$ abdominal aortic aneurysm, CI - confidence interval, CHF - chronic heart failure, CVA - cerebrovascular accident, EVAR - endovascular repair for $A A A, I C U$ - intensive care unit, $H R$ - hazard ratio, OR - odds ratio 


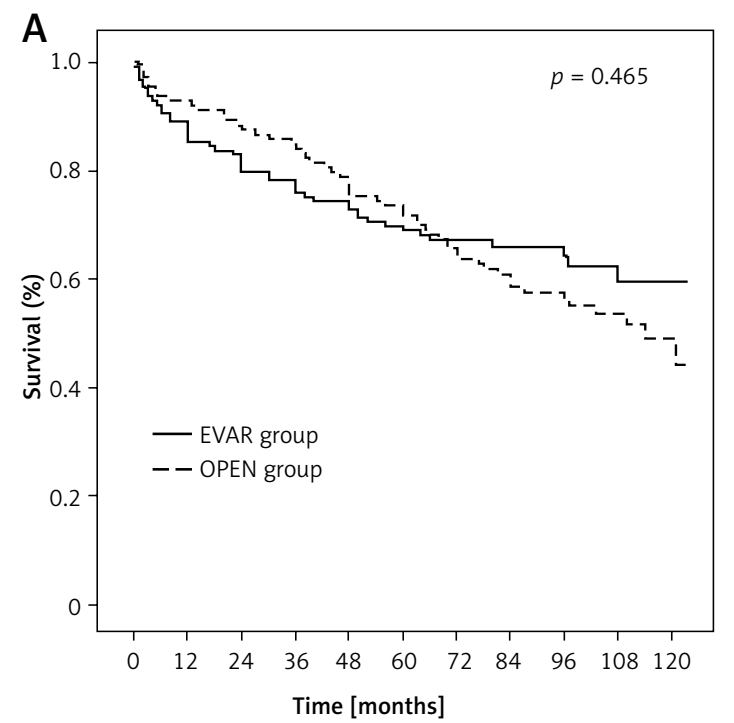

Patient at risk:

$\begin{array}{llllllllllll}\text { EVAR } & 129 & 114 & 106 & 93 & 81 & 69 & 60 & 45 & 33 & 21 & 9\end{array}$

group

$\begin{array}{llllllllllll}\text { OPEN } & 114 & 106 & 90 & 78 & 66 & 54 & 43 & 30 & 18 & 6 & 1\end{array}$
B

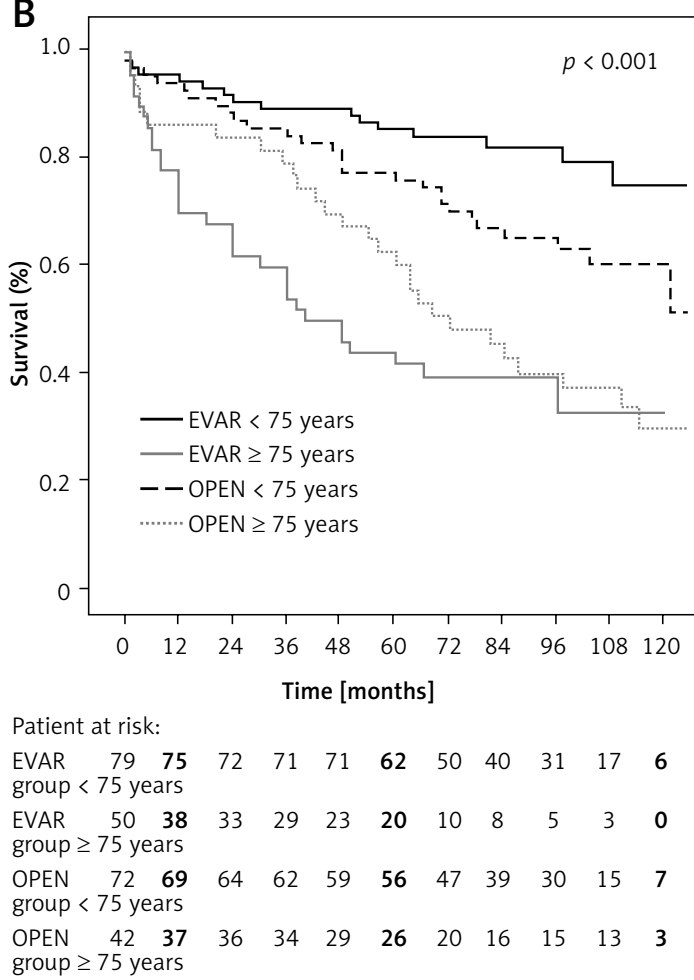

Figure 2. Kaplan-Meier actuarial survival curves for patients undergoing conventional surgical repair (OPEN) and endovascular repair (EVAR) of abdominal aortic aneurysm. A - All patients considered. B - Panel reported differences with reference to age groups

that there was no statistically significant departure from a perfect fit. Similarly, the ROC analysis (AUC of 0.96) revealed excellent discrimination for the multivariable model.

\section{Late outcome}

Two-hundred forty-five patients were discharged alive; two $(0.8 \%)$ patients were lost to follow-up. The remaining 243 patients were followed up for a median of 70 months $\left(25^{\text {th }}\right.$ to $75^{\text {th }}$ percentile, 24 to 101); out of this group, 100 died. Cause of death was AAA-related in 7 cases: specifically, 6 patients (OPEN $n=2$ vs. EVAR $n=4 ; p=0.686)$ died because of a secondary rupture and 1 (OPEN group) died of complications following secondary intervention for thrombosis of the EG. Other causes of death were cerebrovascular accidents $(n=46$, $19 \%)$, cancer $(n=27,11 \%)$, and other causes $(n=$ $20,8 \%)$.

Long-term mortality was similar in the groups $(p=0.465)$ : actuarial survival estimates at 1, 5 and 10 years were $93 \%, 74 \%, 49 \%$ for OPEN procedures, and $89 \%, 69 \%, 59 \%$ for EVAR. A significant difference was observed in younger ( $<75$ years) patients only $(p<0.044)$. Differences were not noted for patients aged $\geq 75$ years $(p=0.216)$ (Figure 2). According to the multivariable Cox analysis, age (HR = 1.09; 95\% Cl: 1.06-1.12), chronic heart failure $(\mathrm{HR}=7.34 ; 95 \% \mathrm{Cl}: 3.24-16.61)$,
ICU admission ( $\mathrm{HR}=2.04 ; 95 \% \mathrm{Cl}: 1.14-3.64)$ and postoperative transfusions $(\mathrm{HR}=1.64 ; 95 \% \mathrm{Cl}$ : 1.04-2.57) were independent predictors for late mortality (Table III).

Late complications occurred in 60 (25\%) patients: 53 (22\%) were procedure-related, whereas 7 (3\%) were ascribed to other causes. The late complication rate was significantly higher in EVAR patients $(p<0.001)$. In particular, the EVAR group experienced a total of 42 procedure-related complications; endoleaks were detected in 23 subjects (type $1 n=10$, type $2 n=11$, and type $3 n=2$ ). In the OPEN group, 11 patients had procedure-related complications: proximal anastomotic pseudoaneurysms $(n=7)$, rupture of secondary aneurysm location $(n=3)$, grade 2 wound infections following the Samson [17] classification $(n=2)$, and graft thrombosis $(n=1)$. Freedom from late complication rates at 1,5 and 10 years were $96 \%$, $92 \%, 86 \%$ for OPEN repair, and $84 \%, 70 \%, 64 \%$ for EVAR (Figure 3).

Over the study period, 38 re-interventions occurred after a median postoperative interval of 14 months ( $25^{\text {th }}$ to $75^{\text {th }}$ percentile, 3 to 41$)$. Ten (26\%) patients required multiple procedures. Emergent re-intervention was performed in 11 (29\%) cases. Re-interventions were endovascular in 23 patients and surgical in 15 . The re-intervention rate was significantly worse in the EVAR group $(p=0.002)$. 
A

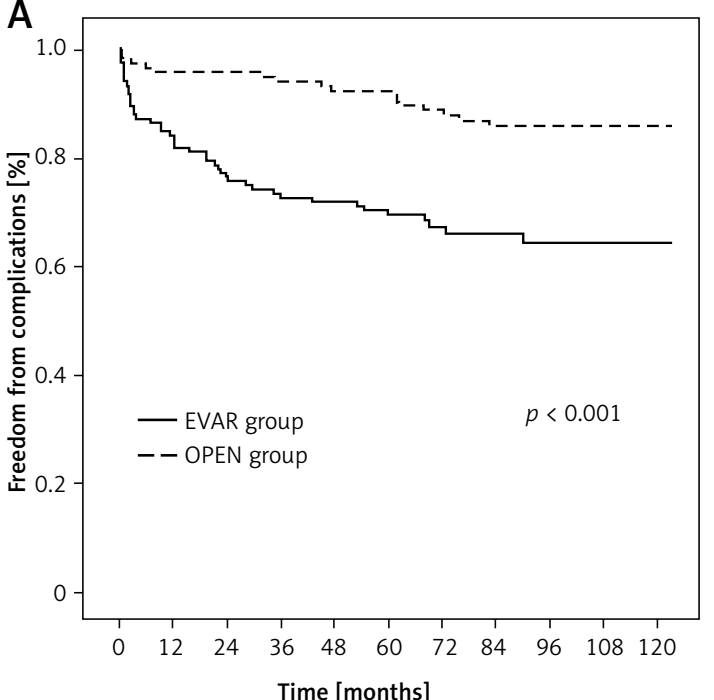

Patient at risk:

$\begin{array}{llllllllllll}\text { EVAR } & 129 & 108 & 99 & 94 & 92 & 85 & 57 & 43 & 30 & 20 & 7\end{array}$

group

OPEN $114 \quad 109109107 \quad 105 \quad 105 \quad 94 \quad 83 \quad 76 \quad 57 \quad 24$

group
B

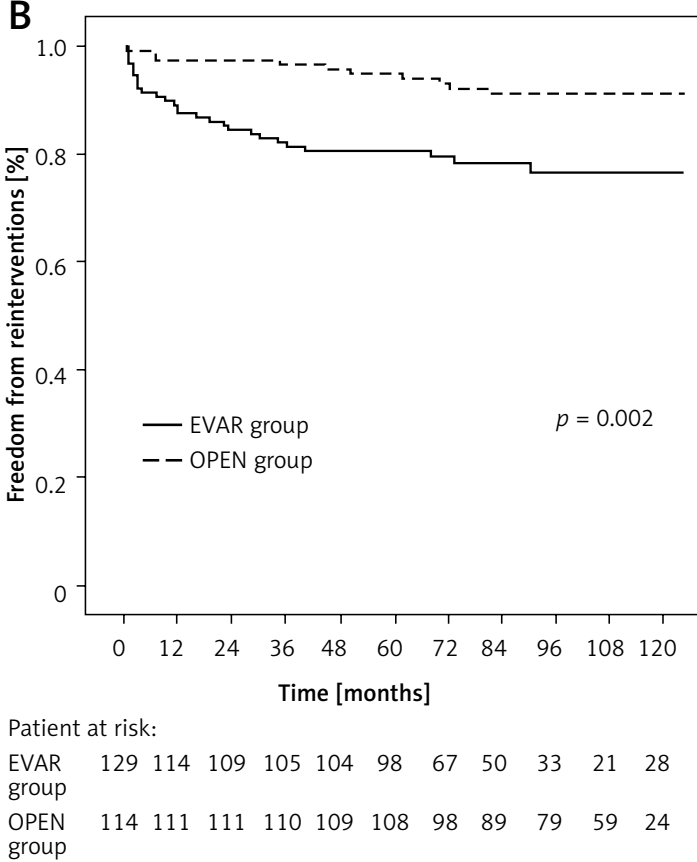

Figure 3. Actuarial freedom from complications and reinterventions between patients undergoing conventional surgical repair (OPEN) and endovascular repair (EVAR) of abdominal aortic aneurysm (EVAR)

Freedom from re-intervention rates at 1,5 and 10 years were $97 \%, 95 \%$, and $91 \%$ for OPEN repair, and $89 \%, 81 \%$, and $77 \%$ for EVAR (Figure 3 ). In the multivariable Cox analysis, independent predictors for re-intervention were the native aneurysm diameter ( $\mathrm{HR}=1.03 ; 95 \% \mathrm{Cl}: 1.01-1.05)$, EVAR procedure $(\mathrm{HR}=2.95 ; 95 \% \mathrm{Cl}: 1.43-6.12)$ and a preoperative history of cerebrovascular accidents $(\mathrm{HR}=$ 2.90; 95\% Cl: 1.39-6.05) (Table III).

\section{Discussion}

The main findings of our study were the confirmation of better overall outcomes for EVAR in the early postoperative period; and in the long-term period, the similar results of mortality and the higher re-intervention rate required after EVAR. These results are consistent with other previous reported papers; nevertheless, our study had the merit of having used an algorithm to obtain two well-matched groups of patients to mitigate potential confounding factors and bias $[8,12,13,19]$.

Previous single-center or randomized clinical trials have shown better efficacy and safety of EVAR in the early postoperative period $[3-5,7,10]$. Few data are available analyzing the long-term outcomes: the results of the EVAR-1 trial highlighted that AAA-related mortality was reduced during the early years after EVAR, but this benefit was completely lost over a median follow-up period of 6 years [10]. Our study was not intended as a randomized clinical trial, but we mitigated the potential bias stemming from the observational nature of our analysis: when comparing the results of the
EVAR-1 trial with the data of our experience, we observe a significant difference in mortality. Survival was not significantly different between the EVAR and OPEN groups in the early postoperative period as well as in the long-term: these data are more surprising if we take into account that we intentionally included urgent repairs for ruptured AAA in the analyses.

Another main finding of the present study is the identification of independent predictors of late mortality. In a previous study on EVAR for high-risk patients, we identified several parameters that adversely affected long-term mortality rate, such as age, ASA score, gender, and native aneurysm diameter [11]. Indubitably, age is an important issue in all surgical interventions. Sicard et al. [12] stated that EVAR can be considered a safe alternative to OPEN repair in octogenarians due to a dramatic reduction in complication and mortality rates. Our experience is in consonance with these data: although we used a different, lowered cut-off (75 years) we noted an advantageous trend to better survival in the early period, as well as non-inferior survival also in the longer run for the older patients. In their report on the first decade of EVAR, Brewster et al. [20] reported similar long-term outcomes, and suggested EVAR as a reasonable alternative to conventional OPEN repair in a broad range of patients with suitable anatomy, including younger and better-risk individuals [20].

Diameter has been identified a significant predictive parameter of long-term mortality. Zarins et al. [21] found that patients with large aneurysms 
$(\geq 6 \mathrm{~cm})$ needed more re-interventions during a 5 -year follow-up, and had a significantly shorter life expectancy than patients with smaller AAAs. Ouriel et al. [22] reported excellent results with EVAR for small AAAs: their mid-term outcomes of large AAAs were associated with increased rates of aneurysm-related death, unrelated death, and rupture. More recently, the CESAR trial showed three important results: no clear advantage between early or delayed EVAR for small AAAs within 36 months of follow-up; most of the small AAAs under surveillance grew and required repair; and notably, $15 \%$ of the AAAs lost EVAR suitability [23]. Our data partly support these results: diameter was noted to be a strong independent marker of long-term mortality, probably because larger AAAs were seen more frequently in older and sicker patients.

The ultimate goal of EVAR procedures is aneurysm exclusion and prevention of rupture [10]. Aneurysm-related mortality is one of the most cited outcomes in published papers. A review of 4291 patients from the EUROSTAR registry by Peppelenbosch et al. [24] identified 34 (0.8\%) late ruptures after EVAR with a mortality rate of $64 \%$. In a recent analysis, Wyss et al. [25] noted 27 ruptures only across the EVAR experience in both EVAR trials 1 and 2 [9, 10, 25, 26]. The paper concluded that this was a low percentage, but also suggested that this small number of ruptures would seem to explain the convergence in aneurysm-related mortality in the recently reported long-term analysis of the EVAR-1 trial [10]. In our study, AAA-related mortality was $2.9 \%$ and rupture was experienced in each group without a significant difference between the groups. It has been supposed that the type of device could have been potentially related to late ruptures $[8,18$, 27]. Wibmer et al. [28] reported 10-year mortality after EVAR of $60 \%$ with $4.6 \%$ AAA-related death, two-fold higher than in EVAR-1 [10]. The authors concluded that the probability of death from late AAA rupture is much lower than the risk of death from other causes during the same period, even in patients treated with first-generation EGs [28]. The experience of Brewster et al. [20] with new generation devices confirmed these observations: they obtained a low rate $(1 \%)$ of late rupture with freedom from AAA-related death of $97 \%$ at 1 year, $96 \%$ at 5 years, and $93 \%$ at 9 years.

The re-intervention rate after EVAR has been confirmed to be high in several studies; most alarmingly, this trend was associated with newer devices too [8, 11, 29, 30]. Becquemin et al. [31] reported a $27 \%$ re-intervention rate at a median follow-up of 18 months, and Sampram et al. [28] estimated a rate of re-intervention of $35 \%$ at 3 years. Endovascular aortic repair patients faced re-inter- ventions more frequently than those operated on conventionally, with a need of redo of $11 \%$ within the first year and $19 \%$ at 5 years. This consistent need for re-intervention after EVAR, coupled with adverse outcomes reported in some papers, led some authors to question the broad application of EVAR [10, 32, 33]. This observation deserves some comments. We should take into account that patients who underwent EVAR probably had a more vigilant follow-up program, particularly in the early phase of the endovascular era. More frequently surveillance was performed at the same center, contrary to what would happen in the case of OPEN repair, therefore potentially underestimating the real incidence of re-intervention. In addition, one should take into account the tendency of some physicians to be more apt to intervene: this could have potentially influenced the higher number of secondary procedures [22, 27]. However, only the long-term analysis of the EVAR-1 trial has ascertained an increased rate of re-intervention for EVAR [10]. It is important to emphasize that also in our experience most of the secondary procedures in the EVAR group were performed endovascularly, and that they did not influence longterm survival. Similarly, in the series of Conrad et al. [34] freedom from aneurysm-related death remained high, as did the overall survival in the redo patients.

The literature is rich in papers quoting re-interventions; unfortunately, not always do they clarify the role of the diameter of the AAA as a predisposing factor for re-intervention [6, 7, 11, 21-24]. In our study, a diameter of more than $55 \mathrm{~mm}$ correlated with increased need for re-intervention. This result has been confirmed in other extensive studies: Sampram et al. [30] found a strong relationship between AAA size and the need for re-intervention, and strongly suggested that unfavorable anatomy, which the literature has demonstrated to have often been associated with larger aneurysm, could be a predictor of re-intervention [30]. Boult et al. [7] found that larger sac size was predictive of the late development of endoleaks requiring reoperation. Our results seem to confirm these data: we found that the maximum AAA diameter could be significantly correlated with higher incidence of unfavorable anatomy, and the combination of a large AAA with hostile anatomy could be significantly associated with increased incidence of re-intervention, and is a useful parameter for better stratification of the risk of re-intervention.

The EVAR-1 trial data showed that the immediate benefits of EVAR are lost in the follow-up along with a greater number of complications, need for re-intervention and consequently an increase inhospital costs [10]. In our study we did not perform 
a cost analysis; nevertheless, re-interventions did not influence negatively the overall mortality rate of EVAR, and we observed a significant reduction of the operation time for EVAR, with an increased ICU admission rate and postoperative transfusion requirement following OPEN repair. Noll et al. [35] performed a cost analysis comparison of EVAR vs. OPEN repairs, with an additional analysis of the costs for the re-interventions. The authors found a higher total cost of $25 \%$ for EVAR; they identified many variables that were associated with increased costs, but the most powerful were the ICU stay and length of hospitalization. These variables could be frequently affected by procedural and/or postoperative complications. Unquestionably, the initial cost of the prosthetic material is much higher in EVAR; however, in our experience EVAR had favorable results in terms of duration of intervention, overall length of hospitalization, as well as in terms of blood transfusions and postoperative ICU resource utilization. It is possible that these data can drastically reduce or obliterate the different economic margin with OPEN repair. These same data are of much interest when paired with those regarding the survival of older patients [33, 36]. Indeed, EVAR had more benefits for older patients, which seemed to be maintained in the long term too. The endovascular approach would thus appear to be the treatment of choice for patients older than 75 years.

Our study has several limitations [5, 23, 30]. It is a single-center observational investigation; selection bias may have been present, although we attempted to mitigate it using a propensity score analysis. However, this process allowed us to identify two (EVAR vs. OPEN) groups of patients, well matched in terms of demographic and morphological characteristics: our experience is a faithful mirror of "real life", no less deserving of importance in terms of "daily" clinical practice.

In conclusion, our study confirms the excellent results of EVAR in terms of safety and significant reduction of early mortality. Age has been confirmed as a significant predictor: patients above 75 years would seem to benefit from EVAR not only in the immediate postoperative period but even in long-term follow-up. Congestive heart disease has been identified as a further marker of long-term mortality: therefore, evaluation of a dedicated predictive score could be of significant utility in identifying those patients at higher operative risk, so that also EVAR could not be justified under very low survival rates in these patients. The diameter of the aneurysm has been the most influential predictor for reoperation: this rate is not irrelevant (19\%) in our experience but most of these "redo" procedures were performed endovascularly and were not significant in terms of mortality.

\section{References}

1. Jones A, Cahill D, Gardham R. Outcome in patients with a large abdominal aortic aneurysm considered unfit for surgery. Br J Surg 1998; 85: 1382-4.

2. Chuter TA, Reilly LM, Faruqi RM, et al. Endovascular aneurysm repair in high-risk patients. J Vasc Surg 2000; 31: 122-33.

3. Sicard GA, Zwolak RM, Sidawy AN, et al. Society for Vascular Surgery Outcomes Committee. Endovascular abdominal aortic aneurysm repair: long-term outcome measures in patients at high-risk for open surgery. J Vasc Surg 2006; 44: 229-36.

4. Matsumura JS, Brewster DC, Makaroun MS, et al. A multicenter controlled clinical trial of open versus endovascular treatment of abdominal aortic aneurysm. J Vasc Surg 2003; 37: 262-71.

5. Alsac JM, Houbballah R, Francis F, et al. Impact of the introduction of endovascular aneurysm repair in highrisk patients on our practice of elective treatment of infrarenal abdominal aortic aneurysms. Ann Vasc Surg 2008; 22: 829-33.

6. Dillavou ED, Muluk SC, Rhee RY, et al. Does hostile neck anatomy preclude successful endovascular aortic aneurysm repair? J Vasc Surg 2003; 38: 657-63.

7. Boult M, Babidge W, Maddern G, et al. On Behalf Of The Audit Reference Group; Audit Reference Group. Predictors of success following endovascular aneurysm repair: mid-term results. Eur J Vasc Endovasc Surg 2006; 31: 123-9.

8. Pulli R, Dorigo W, Marek J, et al. Device specific outcomes after endovascular abdominal aortic aneurysm repair. Ital J Vasc Endovasc Surg 2009; 16: 19-6.

9. EVAR trial participants. Endovascular aneurysm repair and outcome in patients unfit for open repair of abdominal aortic aneurysm (EVAR trial 2): randomised controlled trial. Lancet 2005; 365: 2187-92.

10. United Kingdom EVAR Trial Investigators, Greenhalgh RM, Brown LC, Powell JT, et al. Endovascular versus open repair of abdominal aortic aneurysm. N Engl J Med 2010; 362: 1863-71.

11. Lomazzi C, Mariscalco G, Piffaretti G, et al. Endovascular treatment of elective abdominal aortic aneurysms: independent predictors of early and late mortality. Ann Vasc Surg 2011; 25: 299-305.

12. Sicard GA, Rubin BG, Sanchez LA, et al. Endoluminal graft repair for abdominal aortic aneurysms in high-risk patients and octogenarians: is it better than open repair? Ann Surg 2001; 234: 427-35.

13. Carpenter JP, Baum RA, Barker CF, et al. Durability of benefits of endovascular versus conventional abdominal aortic aneurysm repair. J Vasc Surg 2002; 35: 222-8.

14. Becquemin JP, Pillet JC, Lescalie F, et al. A randomized controlled trial of endovascular aneurysm repair versus open surgery for abdominal aortic aneurysms in low- to moderate-risk patients. J Vasc Surg 2011; 53: 1167-73.

15. Chaikof EL, Fillinger MF, Matsumura JS, et al. Identifying and grading factors that modify the outcome of endovascular aortic aneurysm repair. J Vasc Surg 2002; 35: 1061-6.

16. Chaikof EL, Blankensteijn JD, Harris PL, et al. Ad Hoc Committee for Standardized Reporting Practices in Vascular Surgery of The Society for Vascular Surgery/ American Association for Vascular Surgery. Reporting standards for endovascular aortic aneurysm repair. J Vasc Surg 2002; 35: 1048-60.

17. Rosenbaum PR. Optimal matching for observational studies. J Am Stat Assoc 1989; 84: 1024-32. 
18. Austin PC. Goodness of fit diagnostics fort the propensity score model when estimating treatment effects using covariate adjustment with the propensity score. Pharmacoepidemiol Drug Saf 2008; 17: 1202-17.

19. Abbruzzese TA, Kwolek CJ, Brewster DC, et al. Outcomes following endovascular abdominal aortic aneurysm repair (EVAR): an anatomic and device-specific analysis. J Vasc Surg 2008; 48: 19-28.

20. Brewster DC, Jones JE, Chung TK, et al. Long-term outcomes after endovascular abdominal aortic aneurysm repair: the first decade. Ann Surg 2006; 244: 426-38.

21. Zarins CK, Crabtree T, Bloch DA, et al. Endovascular aneurysm repair at 5 years: does aneurysm diameter predict outcome? J Vasc Surg 2006; 44: 920-9.

22. Ouriel K, Srivastava SD, Sarac TP, et al. Disparate outcome after endovascular treatment of small versus large abdominal aortic aneurysm. J Vasc Surg 2003; 37: 1206-12.

23. Cao P, De Rango P, Verzini F, et al. Comparison of surveillance versus aortic endografting for small aneurysm repair (CAESAR): results from a randomised trial. Eur J Vasc Endovasc Surg 2011; 41: 13-25.

24. Peppelenbosch N, Buth J, Harris PL, et al. Diameter of abdominal aortic aneurysm and outcome of endovascular aneurysm repair: does size matter? A report from EUROSTAR. J Vasc Surg 2004; 39: 288-97.

25. Wyss TR, Brown LC, Powell JT, et al. Rate and predictability of graft rupture after endovascular and open abdominal aortic aneurysm repair: data from the EVAR Trials. Ann Surg 2010; 252: 805-12.

26. Powell JT, Brown LC, Greenhalgh RM, et al. The rupture rate of large abdominal aortic aneurysms: is this modified by anatomical suitability for endovascular repair? Ann Surg 2008; 247: 173-9.

27. Donas KP, Torsello G. Complications and reinterventions after EVAR: are they decreasing in incidence? J Cardiovasc Surg (Torino) 2011; 52: 189-92.

28. Wibmer A, Nolz R, Teufelsbauer $\mathrm{H}$, et al. Complete tenyear follow-up after endovascular abdominal aortic aneurysm repair: survival and causes of death. Eur I Radiol 2012; 81: 1203-6.

29. Ohki T, Veith FJ, Shaw P, et al. Increasing incidence of midterm and long-term complications after endovascular graft repair of abdominal aortic aneurysms: a note of caution based on a 9-year experience. Ann Surg 2001; 234: 323-34.

30. Sampram ES, Karafa MT, Mascha EJ, et al. Nature, frequency, and predictors of secondary procedures after endovascular repair of abdominal aortic aneurysm. J Vasc Surg 2003; 37: 930-7.

31. Becquemin JP, Kelley L, Zubilewicz T, et al. Outcomes of secondary interventions after abdominal aortic aneurysm endovascular repair. J Vasc Surg 2004; 39: 298-305.

32. Pitoulias GA, Schulte S, Donas KP, Horsch S. Secondary endovascular and conversion procedures for failed endovascular abdominal aortic aneurysm repair: can we still be optimistic? Vascular 2009; 17: 15-22.

33. Verzini F, Cao P, Zannetti S, et al. Outcome of abdominal aortic endografting in high-risk patients: a 4-year single-center study. J Endovasc Ther 2002; 9: 736-42.

34. Conrad MF, Adams AB, Guest JM, et al. Secondary intervention after endovascular abdominal aortic aneurysm repair. Ann Surg 2009; 250: 383-9.

35. Noll RE Jr, Tonnessen BH, Mannava K, et al. Long-term postplacement cost after endovascular aneurysm repair. J Vasc Surg 2007; 46: 9-15.
36. Lange C, Leurs LJ, Buth J, et al. Endovascular repair of abdominal aortic aneurysm in octogenarians: an analysis based on EUROSTAR data. J Vasc Surg 2005; 42: 624-30. 